\title{
Knowledge Distribution and the Effect of Design Tools on the Design Process
}

\author{
Mina Tahsiri, Jonathan Hale and Chantelle Niblock \\ University of Nottingham, U.K.
}

This paper compares the cognitive performance of architecture students when designing tasks using one of the three design tools: pencil and paper, software Sketch Up and Rhinoceros 3D. It questions if a design tool can affect when knowledge is generated and used in the duration of design activity. This is explored through a protocol 'think aloud' study for which a new coding scheme was developed. The methodology is grounded on the theory of Distributed Cognition and Zhang and Norman's (1994) method of 'representational analysis', based on which, knowledge is either 'internal' in that it is actively memorized by the designer or is 'external' in that it is implicitly made available via a stimuli like a design tool. Using an Analysis of Variance (ANOVA) test, for the five participants of this study, external knowledge generated significantly earlier on within the process when using Sketch Up compared to the other two tools.

\section{Introduction}

The design process is understood to be a co-evolving process between problem definition and solution generation, taking the designer from an abstract to a more concrete form of thinking $(1,2)$. This indicates that the structure of the task space is not fully defined at the outset of the process and is susceptible to change (3). As Coyne et al (4) state, exploring within a space that is only partially defined is the subject of creativity. In their view, creativity in concerned with a search for the "paraphernalia" that can define that space, which they express to be knowledge. They further explain that a design system's potential for creativity is in its ability to acquire knowledge but also to control its process and change its own structure. In doing so, a design system makes use of its own components, 
knowledge being one of them, to control the operations and implement required changes. As is explained by Edmonds (5), a knowledge-based creative system makes use of knowledge to further revise and refine knowledge. These accounts suggest that knowledge is not only a structural component of the system, but a product of the processes and also an operational tool. Therefore how knowledge is created, particularly in the earlier stages of a design process, can be said to influence the designer's evaluation on the fitness of their ideas as they unfold $(4,6,7)$. Arguably it will also have semantic implications, as it will both affect the issues the designer attends to and the depth and generality of their application $(1,8)$.

The initial stages of a design process are important in cohesively framing the task as it enables deliberation over a wider breadth of issues before committing to the details (9). To ensure this good practice, it is common in design schools to encourage students to spend a good chunk of their time in framing the problem as best as they can, early on in the process. It has been reported that students that succeed in this section are more creative in their productions (1). This approach reinforces a notion that the earlier knowledge is exerted, the more impact it will have on the structure of the task space.

Respectively this paper questions whether a design tool can control the design process by affecting how knowledge is distributed throughout the duration of the design process. It questions where along the process, the mean distribution of knowledge falls if a designer was to conduct a task using one design tool compared to another. It then examines design behaviour under the influence of the design tool that enabled the earlier average occurrence of knowledge.

Given the importance of the topic for design education, five final year architecture students from the University of Nottingham were chosen to participate in a 'think aloud' protocol design experiment. The experiment required each student to conduct three similarly structured tasks, each time with one of the design tools assigned to that task. These design tools were pencil and paper, software SketchUp and Rhinoceros 3D.

\section{Design tools, knowledge and Distribute Cognition}

Besides the on-going debate on what design tools are better suited for nurturing creative thinking of students, the main interest of this paper in questioning the role of design tools arises from the speculation that they can be more than a peripheral aid to cognition (10). In other words, the role of a design tool transcends beyond enabling the representation and communication of ideas formed in the mind into an external and visual format to acquiring epistemic credit (11). This notion is grounded on the 
theory of Distributed Cognition, which defines cognition as a phenomenon extending the boundaries of the skull and the mind, into the environment. Based on this theory, cognition is distributed across people, artefacts and time involved in the execution of a task (12). The manifestation of cognition into the world is therefore a reflection of an aggregating contribution of all its components and not just processes in the 'mind'.

Notably, the accumulated knowledge which the mind holds, is argued by environmental psychologists to be informed by the experience of performances in the real world. Noe (13) asserts that our knowledge, is not only determined by what is done but also our know-how for doing something. This denotes that in thinking, we incorporate both perception of the past and future into the instantaneous present. To explain how future actions can inform present knowledge, Grafton et al's (14) study is relevant. Their study showed that just the naming of the 'use' of a tool has motor valence and effects cognitive processes. Dartnall's (15) philosophical account also helps understand this further. He states that the world sometimes leaks into the mind, in the sense that we can deploy our sensory abilities offline. Therefore designers can structure their task space and predict their course of action by their knowledge of the tool. In this regard Jonson's (16) study demonstrated how the structure in which digitally native designers attend to design differs from its traditional alternative. He reports that these designers use verbalization rather than freehand sketching as a conceptual tool in the early stages of their process.

On the other hand it could also be claimed that the knowledge a designer brings to the process holds a preconception of a manner of performance. Arguably for most designers this would be the knowledge accumulated through conducting design tasks via freehand sketching, particularly since it is the method employed by many design schools in the early years of educating students. In this regard, an alternative explanation could be provided for studies such as that conducted by Bilda et al(17) where they compared the performance of designers when freehand sketching and when blindfolded (using imagery) and concluded that the actual physical activity of drawing made no significant difference in the overall outcome of the process. Similarity in the designers' cognitive performances could in fact be due to deploying their knowledge of freehand sketching both times regardless of using imagery or an actual pencil to design.

Thereby in understanding how a design tool manifests its cognitive role within a process, design has to be regarded as a distributed cognitive task and the role of the design tool has to be incorporated into the scheme by which the process is analysed. 


\section{Methodology}

In acquiring insight into designer's knowledge, it is common in design studies to rely on the designer's verbalization which is the basis of the protocol study methodology. In this methodology, verbal utterances and design actions of structured tasks are segmented and coded based on a coding scheme. The coding scheme provides an ontological perspective of the design process and therefore allows for a specific analysis of cognitive activity $(18,19)$. The 'think aloud' method was chosen over retrospective verbalization to obtain a direct correspondence between thought content (knowledge) and immediate actions.

Presuming that design can be seen as a distributed cognitive activity, the structure of the tasks for this study had to be designed in a way that enables the knowledge provided (through verbalization) to be further categorized into its 'internal' and 'external' components. The internal components are retrieved from internal memory (the mind), in other words what is memorized and the external components from external memory (the design environment), for example through drawing with the design tool. The knowledge the designer manifests through verbalization and/or drawings are an aggregated and abstract representation of processes within both of these task spaces. Figure 1 provides an illustrated diagram of this concept.

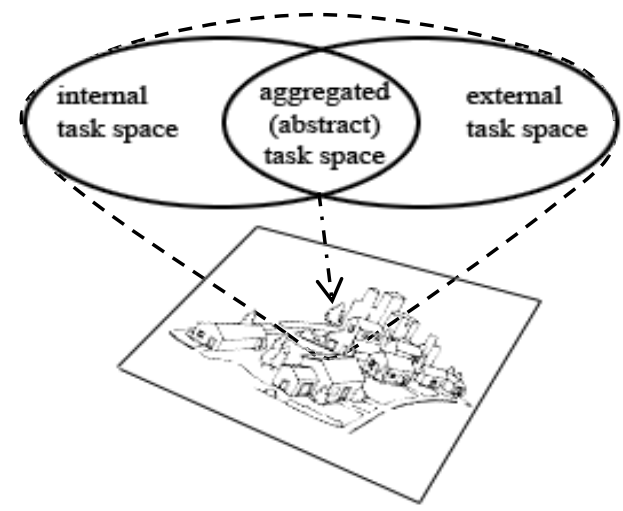

Fig 1. Task spaces within a distributed cognitive system

The necessity of this categorization rises from the fact that analysis of processes conducted at the level of the aggregated task space, cannot fully demonstrate the underlying cognitive differences. In this respect, Zhang and Norman (20) in their study on 'Representations in distributed cognitive task' show how tasks with different internal and external structures can have the same structure when observed at the level of the aggregated task space. They criticize methods used up to that date (1994) in cognitive science studies in analysing distributed cognitive tasks, in that those meth- 
ods, either focused on internal structure, or when taking external structure into account, failed to separate them from internal structure. Therefore they insist on the importance of "decomposing the representation of a task into its internal and external components so that the different functions of internal and external representations can be identified"(20:89).

Respectively studies conducted thus far on the effect of design tools on the design process suggest that the overall quality of the design process, in terms of idea productivity and frequency of attending to design issues is not significantly affected by the design tool used $(17,21)$. Therefore it is suggested that should a design tool affect cognition it will be more on lower level activity such as perception or physical activity rather than conception(22). However it may be that we are facing a similar issue as that identified by Zhang and Norman (20) regarding analysis methods used in cognitive science studies. Similarly it can be argued that in the context of a design experiment, when a designer conducts similar tasks with different design tools, they compensate for the micro internal and external changes in a way that the overall aggregated structure is not significantly affected.

Alternatively Zhang and Norman (20) propose a method called 'representational analysis'. In their account, a task comprises of a series of rules and the structure of the task space represents the relationships between these rules. In the process of solving a problem, the problem solver thus applies these rules to progress towards the solution. To enable use of their method, they structure the tasks of their study so that some of the requirements require active attention and memorization, which they label as "internal rules". These rules are explicitly made available by the conditions of the task. On the other hand some of the requirements are arranged to be implicitly made available by the internal rules or conditions of the task and therefore do not require to be memorized. This category is labelled as the "external rules". In this research a similar approach is employed, based on which a three phase design task and a coding scheme is proposed. The design processes will then be compared and analyzed at the level of the aggregated, internal and external task spaces.

\section{Hypothesis}

Based on what has been discussed thus far, the null hypothesis of this research would be that there is no significant difference between the mean knowledge distributions across the design tools. Should this be approved when analysing distribution at the level of the aggregated task space, it will provide evidence for the veracity of this researcher's scheme and degree of reliability of its method of coding as the result is coherent with results from similar research which suggested that tools do not have a significant 
effect on the overall cognitive activity measures $(17,21)$. On the other hand the expectancy is to find differences at the level of internal and external task spaces. In particular, the research is interested with differences in the mean knowledge distribution in the external task space, where knowledge exclusive to the situation of that particular task is generated and put to use.

\section{The design experiments}

Each participant was required to conduct three tasks, each with its own assigned design tool. To ensure that performances in each task were comparable to one another, the three tasks all had the same structure but represented in different contexts. This means that the requirements in the briefs were the same, but displayed differently so that the designer is under the impression that they are designing a totally different task each time. There was a two week break in between the time each task was taken to ensure that the information learnt in one task does not affect the next. Also, to compensate for the possible affect that the order in which tasks are taken in can have on the results, the order each participant executed the tasks was randomized.

It has been reported that designers' individual approach and their expertise affects the process (23). What is suggested here is that each designer would read and interpret the requirements of the task differently. This calls for a mechanism through which an understanding of the mental model the designer creates from the briefing information is obtained and later used as a reference to analyse the distribution of knowledge in the duration of actual design activity. Therefore each task was comprised of the following three phases:

1- Briefing phase: Participants were asked to watch a short three minutes video clip which display a walk through in the 3D model of the site and explained the brief to them. They were asked to listen and watch carefully. They were then given one minute to recite as much of the information that they had managed to memorize or pick up from the contextual information.

2- Interpretation phase: Here participants were given the written format of the brief with a site plan and three images of the site. They were required to read the brief aloud to provide them a chance to revise the requirements. Repeating the briefing information for a second time is used in similar studies which required participants to enter the design phase without access to the brief (17). According to the Modal model of memory, out of the receive information through our sensory registers, only information which is repeated is processed by memory (short term/long term) other- 
wise it is forgotten (24).The participants were also asked to talk about which information is important to them and why. This enabled an understanding of their rationale in interpreting and analysing, which would make judging the process easier. This phase lasted about five minutes.

Together these two phase enable the participant to construct a mental model which withholds the primary knowledge they will take onto to the design phase.

3-Design phase: In this phase, all the documents except for the plan were taken away from the participants. They were given 40 minutes to develop a concept only using the tool provided and on the $3 \mathrm{D}$ model of the site pre constructed for them. They were specifically asked to plan their time so no process lasts any less than 30 minutes to ensure fairness of comparison. The removal of the written brief and other documents allows the information that is inputted into the process to be controlled.

\section{The tasks}

The tasks asked for a small cultural center on a square shaped site in close proximity to a river and boxed in by east and west neighbors. The freehand sketching process was located in London and asked for the design of an exhibition hall. The Sketch Up process was located in Nottingham and asked for a performing arts center. The Rhinoceros 3D task was located in Leeds and required the design of a public atrium and reading hall. Figure 2 illustrates the 3D models of each site.

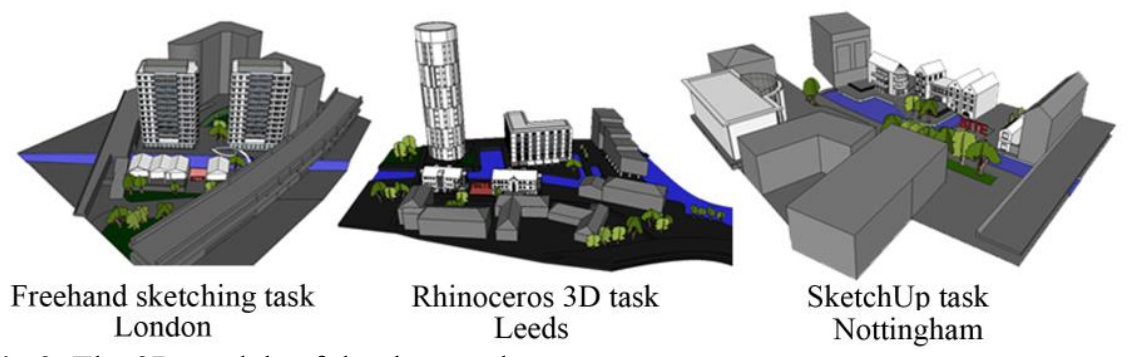

Fig 2. The 3D models of the three tasks.

All tasks were located on sites in the UK, where the participants had more cultural familiarity with. This had proved to be an important factor in a similar pilot study conducted previously, where the task was set in Vietnam and the participants struggled because of the lack of precedent knowledge.

The tasks were designed based on design issues the researchers had known students at the University of Nottingham to have had practice in. Therefore all tasks were centered on incorporating maximum diffused daylight. Each task required one main central space which would cover the en- 
tire ground floor of the site and had to be the most public place of the building. Other complementary smaller spaces that were more private were also needed. A few of these spaces did not need any natural daylighting, such as film and documentary show room for the Rhinoceros 3D task for example. All tasks had the same height restriction. Therefore participants were encouraged to locate the required functions in a creative way that allows each space to receive its required light while maintaining a meaningful narrative in how the building denotes its overall function.

Figure 3 presents an example of the site plans given to the participants. Notably to ensure that the information that can affect external knowledge is similar across the tasks, participants were provided with plans that had been stripped down to some essential information, presented in the same way. This was also the case for the 3D models of the sites. All the architectural elements in the site such as windows had the same details across the tasks.

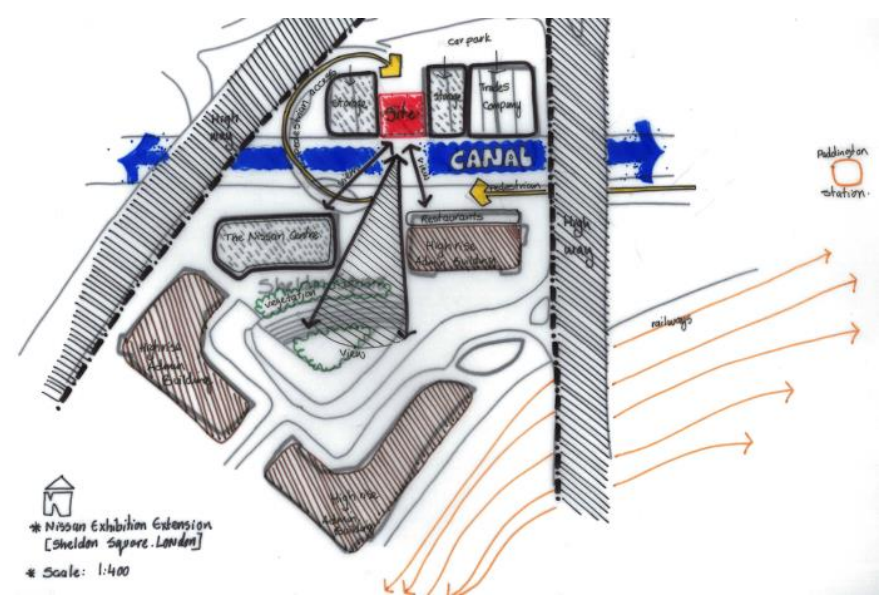

Fig 3. Site plan of the freehand sketching task. Plan redrawn by researchers to ensure equality of information across tasks

\section{Participant recruiting}

A call for participants was sent to final year students in the Diploma Architecture Programme at the University of Nottingham with experience in using the three tools and confidence in developing a concept in those mediums. Out of the volunteers, the suitability of candidates was conferred with two lecturers who knew each student's design skill level. All the students had undergone their undergraduate degree at Nottingham and so came from the same education background. The shortlisted candidates were then invited to an introductory workshop on how to 'think aloud', 
they were given short five minute tasks to accomplish using the software Rhinoceros 3D to practice thinking aloud.

\section{Why the three tools: Pencil on Paper, Sketch Up and Rhinoceros 3D}

In this study, the researchers chose to compare designing with pencil on paper, Sketch Up and Rhinoceros 3D. The latter two are 3D modelling software, which architecture students at the University of Nottingham often use to assist them in developing initial design concepts as an alternative to freehand sketching. These three tools were mainly chosen because of their fundamental differences in how they allow lines and shapes to be manipulated. The speculation is that each method of manipulation will conjure a different mind-set and potential for intervention and this will be the base cause of difference in the distribution of knowledge across the cognitive system.

For example when a designer freehand sketches on paper they inscribe lines into the material surface of the paper. That line is a true, scaled representation of the object it intends to be and its properties remain constant. If they choose to omit the line and rub it out, somehow the indentation will leave its trace behind. With another tool such as Sketch Up, lines are more like narrow sticky tapes. They can be picked up and moved around and if one line is drawn over the other on the same plane, it will dissect the first line into pieces; basically it will stick to it. A continuum of lines leading to a closed shape on one plane will always lead to the appearance of an opaque surface, suddenly making that shape more apparent than any other drawing in the medium. In Rhinoceros 3D however, lines are more like rubber band, they have topological properties and can be stretched, pulled and freely manipulated.

\section{Analysis}

\section{The coding scheme}

Table 1 presents the coding scheme developed for this research. This scheme is knowledge based and process oriented. It describes knowledge as a series of rules applied to the three processes in Asimov's model of 1962, "Analysis", "Synthesis" and "Evaluation". In the proposed scheme, both verbalizations and the design actions are expressed by rule categories. The designer's verbalization is mainly used to understand their rationale and approach, therefore it is seen as a reflection of their "Analysis" processes, which as extensively explained earlier on can be further divided into internal or external rules. 
Table 1 A knowledge based, process oriented coding scheme

\begin{tabular}{|c|c|c|c|}
\hline$\frac{\text { Category }}{\text { Analysis }}$ & Name & \multicolumn{2}{|c|}{ Description } \\
\hline Analysis & $\begin{array}{l}\text { Primary Internal } \\
\text { Rule }\left(\operatorname{IRp}_{\mathrm{x}}\right)\end{array}$ & \multicolumn{2}{|c|}{$\begin{array}{l}\text { Rules created directly from reading the de- } \\
\text { sign brief for the first time, without any } \\
\text { drawing taken out }\end{array}$} \\
\hline & $\begin{array}{l}\text { Generated Internal } \\
\text { Rule }\left(\operatorname{IRg}_{x}\right)\end{array}$ & \multicolumn{2}{|c|}{ Rules created as a result of evaluation } \\
\hline & $\begin{array}{l}\text { Modified Internal } \\
\text { Rule }\left(\operatorname{IRm}_{\mathrm{x}}\right)\end{array}$ & \multicolumn{2}{|c|}{$\begin{array}{l}\text { Rules from the previous design instances } \\
\text { modified as a result of evaluation }\end{array}$} \\
\hline & $\begin{array}{l}\text { Primary Expected } \\
\text { External Rule } \\
\left(\mathrm{ERp}_{\mathrm{x}}\right)\end{array}$ & \multicolumn{2}{|c|}{$\begin{array}{l}\text { Implicit Rules in the design brief, driven } \\
\text { from the primary internal rules. (rules oth- } \\
\text { er than the internal rules which justify the } \\
\text { designers proceeding actions) }\end{array}$} \\
\hline & $\begin{array}{l}\text { Generated Ex- } \\
\text { pected External } \\
\text { Rule }\left(\mathrm{ERg}_{\mathrm{x}}\right)\end{array}$ & \multicolumn{2}{|c|}{$\begin{array}{l}\text { Implicit Rules created as a result of eval- } \\
\text { uation }\end{array}$} \\
\hline & $\begin{array}{l}\text { Modified Expected } \\
\text { External Rule } \\
\left(\mathrm{ERm}_{\mathrm{x}}\right)\end{array}$ & \multicolumn{2}{|c|}{$\begin{array}{l}\text { Implicit rules from the previous design in- } \\
\text { stance modified as a result of evaluation }\end{array}$} \\
\hline \multirow[t]{6}{*}{ Synthesis } & \multirow{5}{*}{$\begin{array}{l}\text { Applied Structural } \\
\text { External Rule } \\
\left(\mathrm{ERs}_{\mathrm{x}}\right)\end{array}$} & \multirow{5}{*}{$\begin{array}{l}\text { Drawing ac- } \\
\text { tions leaving a } \\
\text { mark on the } \\
\text { representational } \\
\text { medium as } \\
\text { rules }\end{array}$} & ERs1-closed lines \\
\hline & & & ERss-Open ended lines $_{2}$ \\
\hline & & & ERs $_{3}$-Appearing lines \\
\hline & & & $\begin{array}{l}\text { ERs4-continuous closed } \\
\text { lines }\end{array}$ \\
\hline & & & $\begin{array}{l}\text { ERs5-continuous open } \\
\text { ended lines }\end{array}$ \\
\hline & $\begin{array}{l}\text { Potential Structural } \\
\text { External Rule }\end{array}$ & \multicolumn{2}{|c|}{$\begin{array}{l}\text { Drawing action or bodily actions (eye } \\
\text { movement/hand movement) not leaving a } \\
\text { mark or actions executed mentally }\end{array}$} \\
\hline \multirow[t]{3}{*}{ Evaluation } & $\begin{array}{l}\text { Transformed Image } \\
\left.\text { evaluation (ERs' }{ }_{1}\right)\end{array}$ & \multicolumn{2}{|c|}{$\begin{array}{l}\text { When no new rule is added to the task } \\
\text { space but an action is executed. These are } \\
\text { evaluation actions occurring [immediately] } \\
\text { after an element has been depicted and do } \\
\text { not involve the drawing of lines. }\end{array}$} \\
\hline & $\begin{array}{l}\text { Primary Image } \\
\text { evaluation }\left(\text { ERs' }_{2}\right)\end{array}$ & \multicolumn{2}{|c|}{$\begin{array}{l}\text { When no new rule is added to the task } \\
\text { space but a drawing action is executed on } \\
\text { the current configuration (design) and usu- } \\
\text { ally happens concurrently with the act of } \\
\text { drawing an element. }\end{array}$} \\
\hline & $\begin{array}{l}\text { Task Space evalua- } \\
\text { tion }\left(\text { ERs's }_{3}\right)\end{array}$ & \multicolumn{2}{|c|}{$\begin{array}{l}\text { When new rules are introduced to the task } \\
\text { space or old rules refined as a result of } \\
\text { transforming actions on the entire configu- } \\
\text { ration (design). }\end{array}$} \\
\hline
\end{tabular}


Coyne et al (4) discuss that a design system consists of five sets of components: "Designs", "Vocabulary of Elements", "Knowledge", "Interpretations" and "Contextual Information". A synthesis of these components can generate new knowledge. Newly generated knowledge needs to be evaluated for its fit in the design. As a result this may induce structural changes in the relationship of other components, which therefore encourages an analysis of the overall structure. In this light Coyne et al (4) indicate that the design system's potential for creativity can be strongly connected to moments of induction of knowledge.

Respectively, in this scheme, the role of the synthesizer is relegated to the design tool in use, in that the tool enables knowledge to be expressed through the tool's specific vocabulary of elements and via the designers' drawing actions. "Evaluation" processes can also be executed through the medium of the tool. An example of which can be rotating a design artefact while thinking. Both "Synthesis" and "Evaluation" actions of such can be regarded as external in that they are readily available and do not require memorization and also structural as they contribute to the generation and modification of knowledge.

The "Synthesis" actions are also further divided based on drawing actions. The intention was to base the division on a language common to drawing regardless of the design tool used. Therefore the most primitive token by which all design action can be describes was chosen: lines (25, 26) The different line type categories in this scheme denote an understanding of the degree of certainty the designer displays while doing that act. For example when a designer is certain about an idea, this is almost always illustrated by a crisp line with two definite end points.

The subdivision of the "Evaluation" actions is temporal. It reflects the scale of evaluation by revealing whether that action occurred concurrently to a drawing action or after. It is also notable that some "Synthesis" actions, namely those conducted in the mind are not codable using this scheme, as analysis must be based on what is observable and represented through verbalization or actual physical activity.

\section{Method of analysis}

For every recorded process a code reference list based on the first two phases of the experiment was made. A partial example of the code reference list for one participant can be seen in Figure 4. This is then used by the coders to interpret which segments embrace which rules. Table 2 shows two coded segments with the code reference list from Figure 4, where the coding of rules $\mathrm{IRg}_{2}$ and $\mathrm{ERg}_{2}$ can be seen in action. Where ever a rule is introduced for the first time, it has been indicated by a bold font. 
In segments where there is more than one rule used they are placed in the order they occur from left to right separated by the $>$ sign.

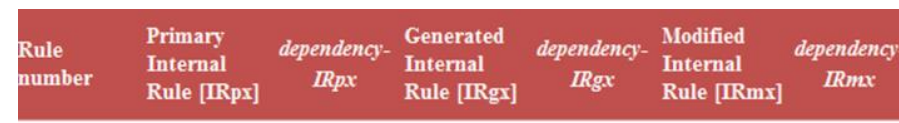

\begin{tabular}{|c|c|c|c|c|c|c|}
\hline 1 & & & $\begin{array}{l}\text { stepping } \\
\text { façade } \\
\text { (bottom to } \\
\text { top) }\end{array}$ & $\begin{array}{l}\text { ERp10+ERp1 } \\
5\end{array}$ & $\begin{array}{l}\text { integration of } \\
\text { stairs in north } \\
\text { façade }\end{array}$ & $\begin{array}{l}\text { IRg4+ERp16 } \\
\text { +ERg4 }\end{array}$ \\
\hline 2 & & & $\begin{array}{l}\text { atrium as } \\
\text { plaza- } \\
\text { continuum of } \\
\text { open public } \\
\text { soace }\end{array}$ & $\begin{array}{l}\text { IRp3+IRp37 } \\
\text { +IRp44+ERp } \\
15\end{array}$ & & \\
\hline $\begin{array}{l}\text { Rule } \\
\text { number }\end{array}$ & $\begin{array}{l}\text { Primary } \\
\text { External } \\
\text { Rule [ERpx] }\end{array}$ & $\begin{array}{l}\text { dependency- } \\
\qquad E R p x\end{array}$ & $\begin{array}{l}\text { Generated } \\
\text { External Rule } \\
\text { [ERgx] }\end{array}$ & $\begin{array}{c}\text { dependency- } \\
E R g x\end{array}$ & $\begin{array}{l}\text { Modified } \\
\text { External } \\
\text { Rule } \\
\text { [ERmx] }\end{array}$ & $\begin{array}{l}\text { dependency- } \\
\qquad E R m x\end{array}$ \\
\hline 1 & $\begin{array}{l}\text { Neighbour 1- } \\
\text { a mix of high } \\
\text { and low rise } \\
\text { commercial } \\
\text { building }\end{array}$ & - & thresholding & IRp15+IRg1 & $\begin{array}{l}\text { use of } \\
\text { structure as } \\
\text { framing } \\
\text { object }\end{array}$ & ERp16+ERg1 \\
\hline 2 & $\begin{array}{l}\text { Neighbour 2- } \\
\text { railways }\end{array}$ & - & $\begin{array}{l}\text { atrium as } \\
\text { central feature }\end{array}$ & IRp37+IRg3 & $\begin{array}{l}\text { atrium as an } \\
\text { open habitual } \\
\text { space }\end{array}$ & IRg2+ERg2 \\
\hline
\end{tabular}

Fig 4. An example of part of the code reference list for one participant

Table 2 An example of two coded segments

\begin{tabular}{|c|c|c|c|c|}
\hline Verbal utterance & 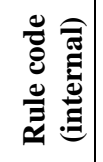 & 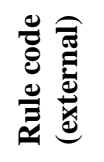 & 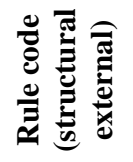 & 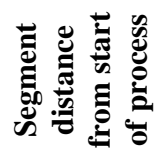 \\
\hline $\begin{array}{l}\text { or maybe or maybe it could become a multi- } \\
\text { functional space. [moves cursor to explain] }\end{array}$ & - & $\operatorname{Erg}_{1}$ & ERs'3 & 18 \\
\hline $\begin{array}{l}\text { And erm, then I need to create this vertical } \\
\text { link between the atrium and library, and } \\
\text { er,[moves cursor as if drawing, zooming in } \\
\text { and out] let me just, because it's all square } \\
\text { and erm, and we have this solid wall, I will } \\
\text { place them,[goes to plan view port and moves } \\
\text { cursor to explain] the stairs in the, sort of } \\
\text { middle, but not exactly central but the, in the } \\
\text { middle of this solid wall. Just see what hap- } \\
\text { pens, it's just kind of idea of, I guess right } \\
\text { now. }\end{array}$ & $\begin{array}{l}\mathbf{I R g}_{2} \\
\text { IIR } \\
\mathbf{p}_{34}\end{array}$ & $\begin{array}{c}\text { ERp }_{6}> \\
\text { ERg }_{2}\end{array}$ & $\begin{array}{c}\text { ERs'3> } \\
\text { ERs'3 }\end{array}$ & 19 \\
\hline
\end{tabular}


For the comparison of knowledge distribution, the variable was the number of segments each rule is distanced from the beginning of the design stage. The means and standard deviations of the distributions for each participant's tasks were calculated regardless of the rule type, for all three task spaces: internal, external and the aggregated. However since segments were not of equal time length across participants, the data necessary for analysis (the mean and standard deviations) was re-expressed in terms of the percentage of time passed when they occurred in their process. The aim was to underpin possible significant difference in the mean knowledge distribution based on the design tool used. Therefore a one-way ANOVA (Analysis of Variance) test using IBM SPSS 21 was utilized here.

To not violate the assumptions of the ANOVA test, gaining assurance of the normality of the data was required. The Shapiro-Wilk test was used for this purpose. Also the homogeneity of variance of the means had to be checked for which Levene's test(27) was used. Where the ANOVA indicates a significant difference at a level of $\alpha=0.05$, this was followed up by a pairwise comparison applied through the LSD (Least Significant Difference) post-hoc test to determine where within the data the difference lies.

Analysis was conducted at these levels: aggregated task space, Internal task space, External task space and External task space (new rules only)

It is also notable that due to a small study group (15 experiments) the intention was not to generalize findings from this study. The ANOVA was utilized only to show that a difference in the means of distribution, is a difference that is considerable and worth further investigation.

\section{Results}

Figure 5 displays the mean knowledge distribution of the 15 processes at the level of the aggregated task space, where the error bars represent the standard deviation.

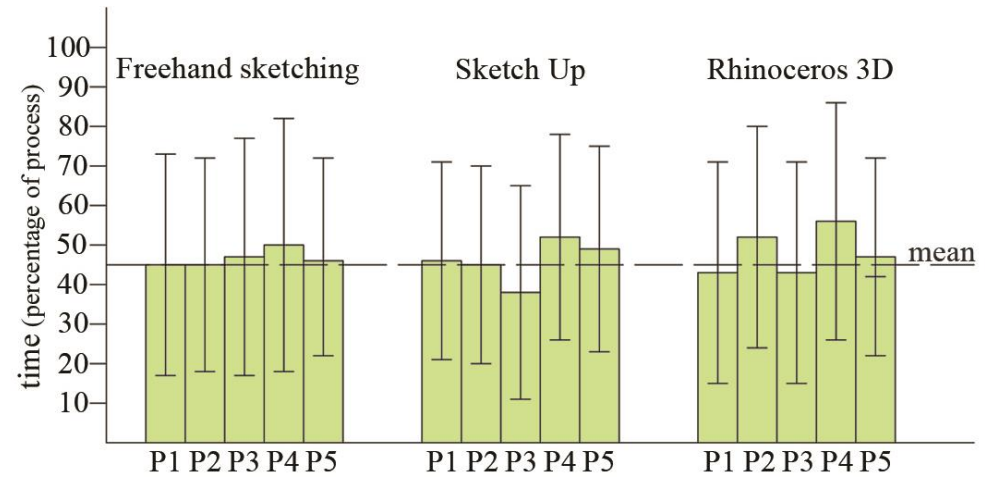

Fig 5. Mean knowledge distribution and standard deviation of design processes 
On average, in all processes regardless of the tool used or the participants' individual approaches, the mean knowledge distribution occurs just under half way within the process, at $45 \%$. Each process on average also held a standard deviation of $28 \%$. This fairly wide clustering of knowledge around the mean indicates that participants are cognitively active throughout the process. A closer look at the distributions showed that all throughout the process, the participants managed to generate new knowledge. Therefore it was not just the case that the creation of new knowledge were dedicated to a specific interval within the process. This alternation between reuse and generation of knowledge would thereby suggest that an executed action through the use of the design tool has a contributing role.

Based on the Shapiro-Wilk test, processes conducted with each tool represent a normal population (null hypothesis rejected since $p$ value $>\alpha=0.05$ for each tool. The $p$ values are; freehand sketching:0.71, SketchUp:0.80, Rhinoceros 3D:0.37). The 15 processes also indicate a homogenous population using Levene's test (null hypothesis rejected with a $p$ value of 0.34 ). Having passed these two tests, the ANOVA can be applied.

Studying Figure 5, the effect of the participants' individual differences is also more apparent in tasks executed using the 3D modelling software, whereas in the freehand sketching process their performance in terms of knowledge distribution is very similar. However the ANOVA shows the differences at this level of analysis, to be insignificance (null hypothesis accepted with a $p$ value of 0.75 ). Therefore the tool has no significant effect on the overall (aggregated) cognitive process.

However it was still of interest to this study to understand why the participants' performance in freehand sketching were more similar and consistent compared to the other two. Therefore, three categories of their design actions were further observed; ERs 1 -the drawing of closed lines, which displays certainty in the participants' decision making; ERs' ${ }_{2-}$ evaluation concurrent to a drawing action, which displays constant consideration of small scale spatial relation; ERs' ${ }_{3}$-evalaution of the overall task space, which represents consideration of fitness of ideas with the requirements and large scale spatial relation. Figure 6, shows that all participants, were noticeably evaluating their concurrent actions more in the free hand sketching process. They were also excessively engaged with drawing actions using this tool. In the case of the 3D modelling tools, there is proportionally more evaluation of the task space compared to the amount of lines drawn.

Based on the observation of the processes, in the freehand sketching process, the participants are more dependent on the tool to inform their external knowledge. They break the problem into smaller chunks and explore 


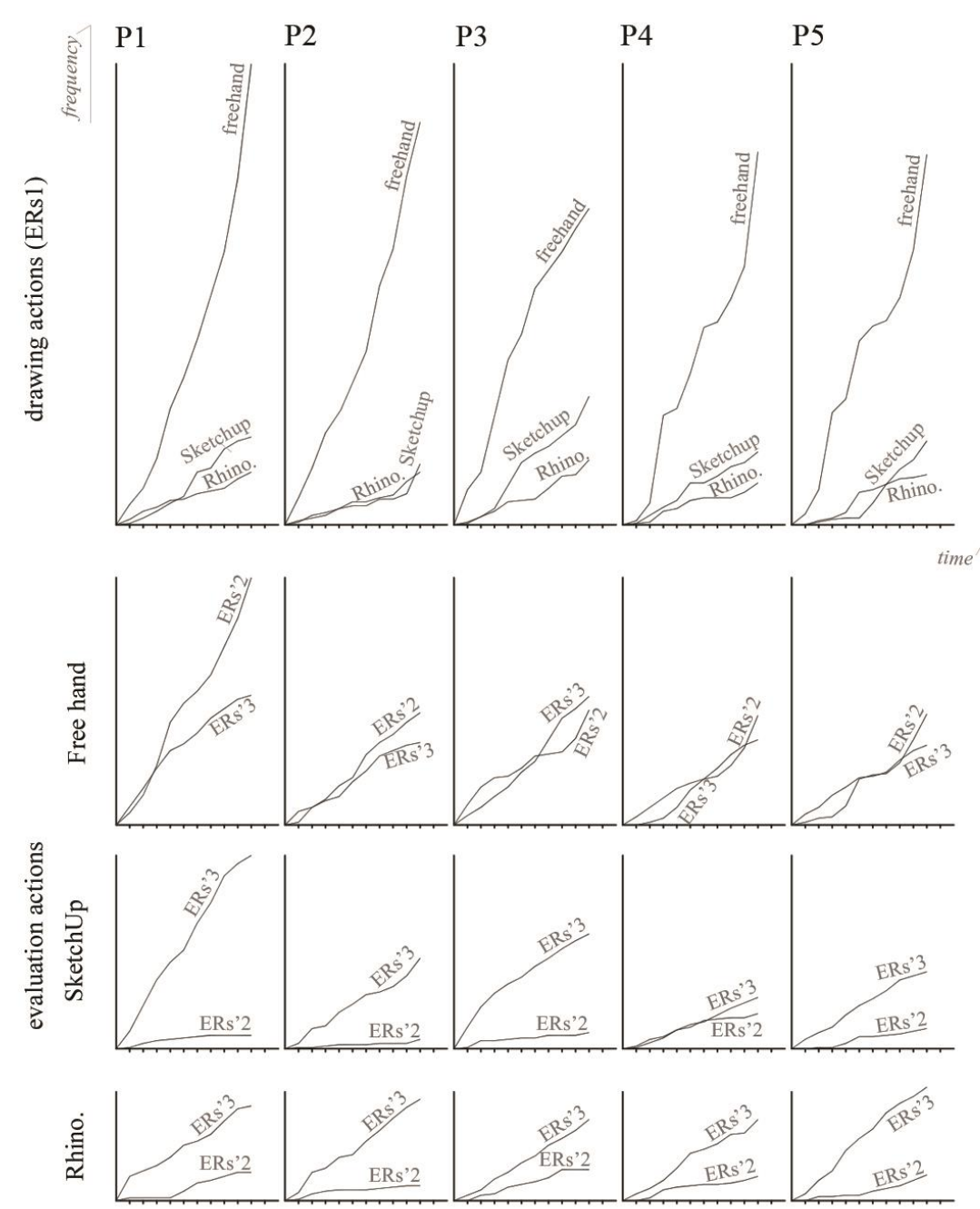

Fig 6. The participants' frequency of drawing and evaluating actions

small scale options by excessive drawing (rapidly re-drawing over an already depicted shape) without simultaneously being concerned about the effects of the actions on the overall configuration. However using the other tools, constant concern about the overall configuration (which is encouraged through the possibility of zooming in and out for example), means that each drawn line plays more of an importance within the overall picture of the design. Therefore the participant only commits to drawing that line when assurance of its necessity is reached. In this sense, as each drawing action using these tools would bear more semantic value, it can also dramatically change the structure of the design process compared to when 
freehand sketching. As a result more divergence between participants using the 3D modelling software can be expected.

The first part of the study shows that regardless of differences in actions, participants had regulated their processes so that the overall mean knowledge distributions were not affected. The second part of the study requires looking at whether the effect of these differences can be picked up when analysing distributions within internal and external task spaces. Figure 7 illustrates the findings. When comparing the mean knowledge distribution of all rules in the internal and external task spaces the ANOVA indicated no significance difference (internal task space- $p$ value: 0.39, external task space- $p$ value: 0.37 ) However when we conducted a filtered analysis on the moments new rules were introduced, the differences between the mean knowledge distribution across the two task spaces for each process were more dramatic (the hatched areas in Figure 7).

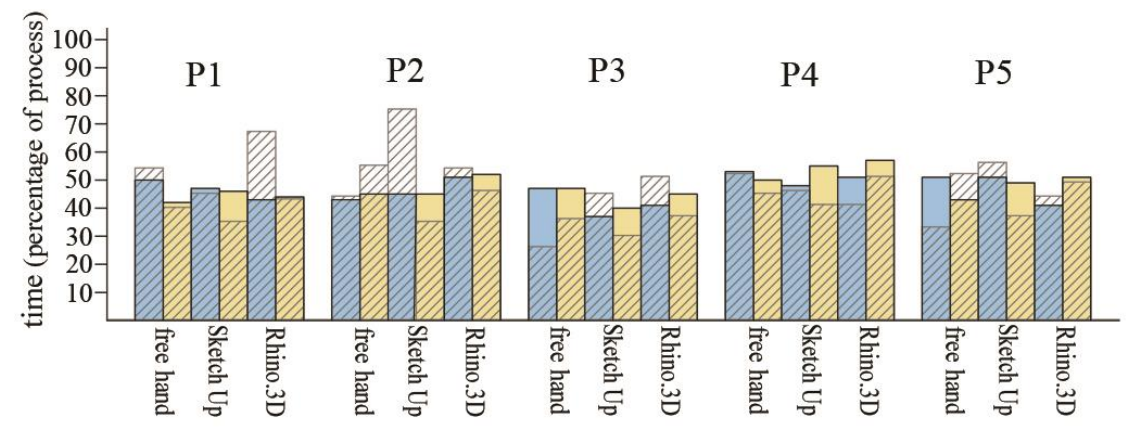

Fig 7. Comparing mean knowledge distribution across internal and external task spaces. (blue: internal task space; yellow: external task space; hatched: mean distribution of new rule introduction)

In analysing the differences in the distribution of new rules across the task spaces, there was a case of violation of normality for the mean knowledge distribution of new internal rules, so we further looked to test the ANOVA for differences in the mean knowledge distribution of new external rules only. Here, it was shown that there is a significant difference $(p$ value: 0.04$)$. The LSD post-hoc test, showed this difference to lie within the mean knowledge distribution when comparing freehand sketching to Sketch Up, and Rhinoceros 3D to Sketch Up but not in between Rhinoceros 3D and freehand sketching, which is reflected in Figure 8.

This would imply that in design, the elements external to the task such as the design tool and the environment it creates for design do affect the process. For these participants, the fact that Sketch Up significantly entices external knowledge generation (which was discussed to be dependent on 
the tool in the case of this study) early on in the process, together with the semantics that as described drawing actions bear, means that this tool has had more of an impact of the structuring of the process and how ideas are defined.

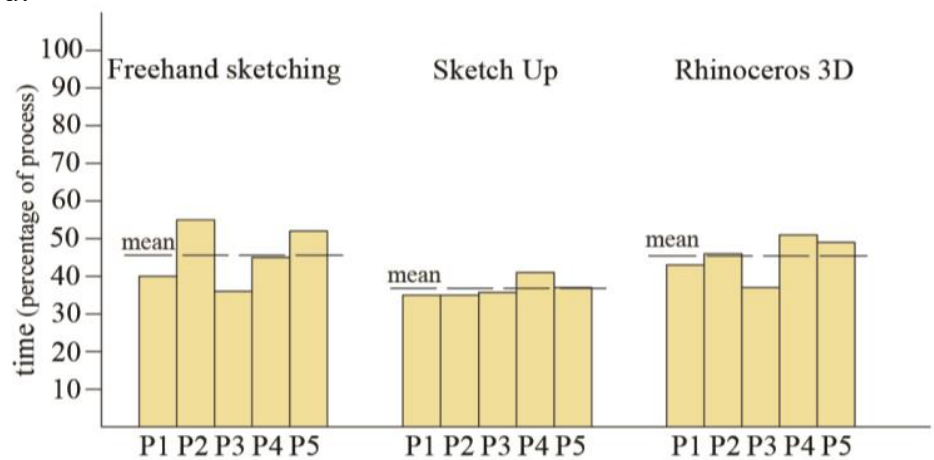

Fig 8.Comparing differences for the mean knowledge distributions of new external rules

\section{Discussion}

Based on the results, the question of interest for further discussion is on how design behaviour using Sketch Up in the earlier stages of the process differs from that conducted using the other two tools. Achieving a neat design- that is one where drawings do not lead to frustrating issues, such as surfaces not forming in the same plane or objects becoming integrated into other objects unintentionally- using Sketch Up requires attention and planning. Using this tool- more than the other tools- the designer is encouraged to break down the process based on the affordance of the workspace, because the cost of errors can be quite high. Errors impose hindrance in the creative process and the sequential flow of ideas (28). Retrieving from a mistake in free hand sketching requires less effort and time and therefore an inappropriate decision would not affect the process as much as it would using Sketch Up.

Therefore as observed in this study, to ensure sound decisions, the participants relied heavily on contextual information made available to them when using Sketch Up. Objects were often created as visual references to help the participant not diverge from the domain which they believed will yield in their desired design concept. An example of this can be seen in participant Jon's process in figure 9.

What is postulated in this paper is that how the visual information at each moment is framed also affects the process. In Sketch Up the designer works on one 3D canvas, constantly exposed to all information. In freehand sketching, only so much can be depicted in each perspective drawing 

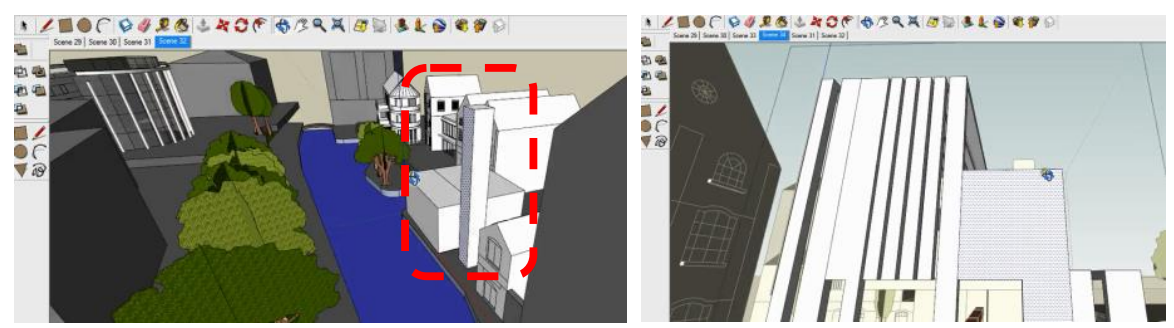

Fig 9. Left: An example of creating visual references in Sketch Up. Right: zooming in, in Sketch Up to reduce presence of contextual information in frame.

or on one canvas. Therefore the designer can assess and read the information more effectively because the load has been broken down. Some times it is easier to solve superficial and present issues first before attending to more complex issues. Working on one canvas, holds the designer back from focusing on delving into the deeper issues as there are many trivial issues that can be attended to. There is a tendency in designers to shift between frames or to minimize the frame so that it can help them focus on a small cluster of information at each given time. In this respect Rhinoceros 3D's provision of four view ports is more effective than Sketch Up. Figure 9 shows instances when Jon has zoomed in to exclude contextual information in Sketch Up so as to focus on a specific issue.

In the case of this study, interestingly new knowledge distribution for both free hand sketching and Rhinoceros 3D process was the same. However as discussed, these tools provide different workspaces and encourage different methods of manipulation. This suggests that different methods of manipulation can be isomorphs of one another, meaning that we do not necessarily require the assistance of intuitive or physically tangible tools such as pen and pencil to aid creative thinking. Therefore it can be concluded that tools have a cognitive role in the design process which goes beyond the physical nature of the tool. The change they apply is in the breaking down of the process and affordance for effectively handling information.

\section{Conclusion}

This paper looked at how design processes can be affected by the tools used to execute them. The study was limited to a small sample size. Therefore, the findings are not considered to be truly representative of what happens in design processes. However, it did manage to show the necessity of decomposing cognitive activity into its internal and external components for an effective study of differences in processes under the influence of varying external conditions. 
A new coding scheme was also introduced based on the theory of Distributed Cognition that can be used in protocol design studies, where the role of tools is incorporated into the structure of the scheme. The scheme assisted in categorizing the knowledge used in the process as a series of rules. Studying the distribution of knowledge (rules), the results suggested there to be no significant difference between the tools when processes are compared at level of the aggregated task space. Research in this field, is currently focused on analysis at this level. However a significant difference was in how the tools had affected the introduction of new knowledge within the external task space.

The main argument here was that the reason for such outcomes, surpasses differences in the physicality of the tools. The tools afford different controlling qualities in the decision making structure and incorporation of information available to the designer, which affects creativity by determining when and how new knowledge is introduced to the process. Acknowledging this conclusion, we can investigate how different methods of manipulation can result in isomorphs for a creative cognitive system.

\section{References}

1. Dorst K, Cross N (2001) Creativity in the design process: Co-evolution of problem-solution. Design Studies 22(5):425-437

2. Maher ML, Poon J (1995) Co-evolution of the fitness function and design solution for design exploration.in Evolutionary Computation, 1995 IEEE Conference on 1:240

3. Gero JS, Fujii H (2000) A computational framework for concept formation for a situated design agent. Knowledge-Based Systems 13(6):361-368

4. Coyne RD, Rosenman MA, Radford AD, et al. (1987) Innovation and creativity in knowledge based CAD. Expert Systems in Computer Aided Design 435-465

5. Edmonds EA (1993) Knowledge-based systems for creativity. Modelling Creativity \& Knowledge-based Creative Design 259-271

6. Coyne RD, Gero JS (1986) Semantics and the organization of knowledge in design. Design Computing 1(1):68-89

7. Goel V, Pirolli P (1992) The structure of design problem spaces. Cognitive Science 16(3):395-429

8. Suwa M, Gero JS, Purcell T (1998) Macroscopic analysis of design processes based on a scheme for coding designer's cognitive actions. Design Studies 19(4):455-483

9. Dinar M, Shah JJ, Langley P, et al. (2011) A structure for representing problem formulation in design. in DS 68-6: Proceedings of the $18^{\text {th }}$ International Conference on Engineering Design, ICED11 . Impacting Society through Engineering Design, Vol 6:Design Information and Knowledge. Copenhagen, Denmark 
10. Baber C, Parekh M, Cengiz TG (2014) Tool use as distributed cognition: how tools help, hinder and define manual skills. Frontiers in psychology 5:1-14

11. Clark A, Chalmers D (1998) The extended mind. Analysis 58(1):7-19

12. Hollan J, Hutchins E, Kirsh D (2000) Distributed Cognition: Toward a New Foundation for Human-computer Interaction Research. ACM Transactions on Computer.-Human Interaction (TOCHI) 7(2):174-196

13. Noe A (2006) Action in perception. The MIT Press, Cambridge: Mass.

14. Grafton ST, Fadiga L, Arbib MA, et al. (1997) Premotor cortex activation during observation and naming of familiar tools. Neuroimage 6(4):231-236

15. Dartnall T (2005) Does the world leak into the mind? Active externalism,"Internalism" and epistemology. Cognitive Science 29(1):135-143

16. Jonson B (2005) Design ideation: the conceptual sketch in the digital age. Design Studies 26(6):613-624

17. Bilda Z, Gero JS, Purcell T (2006) To sketch or not to sketch? That is the question. Design Studies 27(5):587-613

18. Dickson J, Mclennan J, Omodei MM (2000) Effects of concurrent verbalization on a time-critical, dynamic decision making task. The Journal of General Psychology 127(2):217-228

19. Ericsson KA, Simon HA (1993) Protocol analysis verbal reports as data. The MIT Press, Cambridge: Mass.

20. Zhang J, Norman DA (1994) Representations in distributed cognitive tasks. Cognitive Science 18(1):87-122

21. Yu R, Gero JS, Gu N (2013) Impact of using rule algorithms on designers' behavior in a parametric design environment: Preliminary results from a pilot study. Global Design and Local Materialization 13-22

22. Tang HH, Gero JS (2001) Roles of knowledge while designing and their implications for CAAD. in Proceedings of CAADRIA 1:81-89

23. Eisentraut R (1999) Styles of problem solving and their influence on the design process. Design Studies 20(5):431-437

24. Gazzaniga M, Ivry RB, Mangun GR (2014) Cognitive neuroscience: The biology of the mind. W. W. Norton \& Company Ltd, New York

25. Arnheim R (1977) The Dynamics of Architectural Form: Based on the 1975 Mary Duke Biddle Lectures at the Cooper Union. University of California Press, London

26. Mitchell WJ (1990) The logic of architecture: Design, computation and cognition. The MIT Press, Cambridge: Mass.

27. Spiegel MR, Stephens LJ (2008) Schaum's outline of statistics. 4th ed. McGraw-Hill

28. Nijstad BA, Stroebe W, Lodewijkx HF (2003) Production blocking and idea generation: Does blockage interfere with cognitive processes? Journal of Experimental Social Psychology 39(6):531-548 